\title{
A Latent Class Conjoint Analysis for the Administrative Clerk figure: insights from ELECTUS
}

\author{
Blinded version
}

Received: date / Accepted: date

\begin{abstract}
ELECTUS is a multi-centre research project with the aim to stabilize the relationship between universities and companies. Through this project, it is possible to acquire information about procedures for the entrepreneurs' choice in the recruitment process of the new graduates. Using a CAWI survey, Lombardy companies with at least 15 employees were asked to manifest their preferences choosing among hypothetical profiles of new graduates with different competencies each other. In this study a Conjoint Analysis is carried out to evaluate which characteristics of a graduate's profile the employers prefer for a potential candidate in the job position of Administrative Clerk. More specifically, in order to detect if there exist subgroups of employers having homogeneous preferences about such characteristics, a Latent Class Metric Conjoint Analysis is employed.
\end{abstract}

Keywords Labour Market · Latent Class Models · Conjoint Analysis · Electus

\section{Introduction}

During last years, the economic crisis exhibits effects about performances in business and particularly in the employment in all European countries. The impact of this crisis struck weaker segments of the labour market, in detail young person and people with less work experience [19]. Most of the times, there is no possibility for turnover, so the younger people are not able to access the labour market. In Italy, from 2007 over 2014, young unemployment rate (15-24 years) increased from $20.4 \%$ to $44.7 \%$ [9]. This increase of the unemployment rate has some immediate effects: it disheartens young people to search for a job and it convinces them to take shelter in academic studies in order to find the right key to open the door of the labour market. The

Address(es) of author(s) should be given 
worst situation is represented by whom give up in the job research and in the educational studies, growing up the NEET (Not engaged in Education, Employment or Training) group. According to an European strategy, solving the problem of unemployment represents a fundamental aspect for the integration of young people in the labour force. This represents also one of the objectives of the Horizon 2020 program [4]. The universities can play a central role in this sense, being at the centre of the triangle composed by Education, Research and Innovation. They could realize this aim building some tools connecting the labour market and the students for every study level from the beginning to the end of university path of the student. This could reduce the time required for contact between the job seeker and the firm, as a search channel efficiency indicator [11]. In relation to the labour market, it reveals evident how the other side of the phenomena is represented by the companies and their expectations about the possibility for a new hiring. It appears useful to carry out information from the companies' point of view obtaining a deep analysis about what they are looking for. For this reason, in 2014 the University of Padua presented the multi-centre ELECTUS, a research project involving several Italian universities. The project wanted to acquire information about the procedures of recruitment of new graduates. The enterprises were asked to manifest the preferences on several profiles of new graduates for several positions. The survey was devised and structured by the use of a particularly complex conjoint experiment.

The aim of this paper is to carry out a segmentation analysis [26] of employers' preferences for graduates' profiles evaluated as candidates in a job position by using a Latent Class Metric Conjoint Analysis (LCMCA) [3]. Specifically, the analysis aims to detect the existence of unobserved subgroups of employers having homogeneous preferences about graduates' characteristics and to identify the covariates which may affect such evaluations for each subgroup.

The paper is organized as follows. Section 2 describes the context of the research and some descriptive statistics that characterize the analysed data. Section 3 presents the figure of Administration Clerk. Section 4 introduces Latent Class Metric Conjoint Analysis. Section 5 presents the results of the LCMCA. Finally, Section 6 is reserved to discussion and final remarks.

\section{The ELECTUS project}

In a perspective of synergy between education and labour market, a possible solution is represented by some multi-centre research as ELECTUS, an acronym standing for Education-for-Labour Elicitation from Companies' Attitudes towards University Studies, a research project involving several Italian universities [5]. ELECTUS was created with the objective to measure the job offer submitting a survey to the companies and concerns the comprehension policies about relationships between the enterprises and universities, with reference to the labour market for the new graduates. The questionnaire contains two macro-sections, in the first part the entrepreneurs are asked to choose and 
rank four possible profiles of new graduates for five different job vacancies. In the second part the entrepreneurs are asked about their socio-demographic features. The survey was conducted in 2015 at the University of Milano-Bicocca using CAWI technique. Data were collected using a software program called Sawtooth [18]. In order to measure entrepreneurs' preferences, a conjoint experiment was conducted in these terms [5]:

- First of all, each entrepreneur was asked to to select the best among four hypothetical profiles of candidates for a junior job position. The four profiles were presented with one vignette describing, in random order, the four profiles. The respondent was asked to pinpoint the most preferred one as the closest figure to the ideal candidate for a given position. The question was 'Imagine that you are interested in recruiting a person for the position of (). Which one out the four profiles that appear in the screen would you invite for a job interview, it being the closest to your ideal candidate?'. Obviously, the choice is related to the moment of pre-selection of candidates (of course before the job interview) and it is based on CV.

- In a second moment, questions on the features qualifying the choice were also posed. Among the others, one question was to mark each profile on a scale of 1 to 10 .

Five $^{1}$ job positions were considered as vacant: Administrative Clerk, Marketing Assistant, Human Resource Assistant, Customer Relationships Manager and Information Communication Technology Professional (system analyst). The candidates' profile are characterized by six attributes:

- Field of Study with 10 levels (Philosophy and Literature, Educational Sciences, Political Sciences/ Sociology, Economics, Law, Statistics, Industrial Engineering, Mathematics/Computer sciences, Psychology, Foreign Languages);

- Degree Mark with 3 levels (Low, Medium, High);

- Degree Level with 2 levels (Bachelor, Master);

- English Knowledge with 2 levels (Suitable for communication with foreigners, Inadequate for communication with foreigners);

- Relevant Work Experience with 4 levels (No experience at all, Internship during or after completion of university studies, Discontinuous or occasional work during university studies, One year or more of regular work);

- Willigness to Travel on Business with 3 levels (Unwilling to travel on business, Willing to travel on business only for short periods, Willing to travel on business even for long periods).

The attributes used to describe the possible candidates were selected after a literature review and thanks to the elicitation of experts opinions through a focus group at the University of Padua. The profiles submitted to the entrepreneurs' attention are simply a 'sample' of the large set taught in a general university. Effectively, this survey could aim to consider the usefulness of disciplines taught at the universities respect to the five job positions and the

1 The paper focus the attention on Administration Clerk. 
entrepreneurs' preferences. The combinations for all the alternatives provided by a full factorial fashion were numerous that it was necessary to reduce the possibilities using an ad-hoc fractional factorial design. The universe of all possible alternatives was arranged and a random sample of four was administered to each respondent. This way, it was obtained from respondents enough information on the entire spectrum of attribute levels. An individual questionnaire was generated according to the following criteria:

- Minimum overlap criterion: each attribute level should have appeared as few times as possible in a single questionnaire;

- Level balance criterion: each attribute level should have appeared approximately an equal number of times both within a single questionnaire and in the whole sample

- Orthogonality criterion: attribute levels should have been defined independently of other attribute levels, so that the effect of each attribute level could be measured independently of the others.

At the end, given the assumption of non-significance of higher-order effects, the experimental design was both orthogonal and balanced [10]. The experimental design was realised by the Sawtooth program itself.

As far as the Milano-Bicocca research unit is concerned, interviewees were representatives of companies registered on the Portal of Almalaurea for recruitment and linkage, limited to the university site. The population of companies targeted was composed by 4.183 potential recruiters. Companies received a first e-mail inviting to complete the survey. If they did not answer after the first attempt, they were solicited to fill in the questionnaire for three times, once a week. After these attempts, final respondents were 471. Companies profile shows that they were in prevalence sized with 15-49 employers (52\%), followed by sized, 50-249 employees enterprises (25.6\%) and (22.4\%) by sized at least 250 companies. The most represented activity sectors were services to the industry $(62.1 \%)$, services to the person or the family $(16.2 \%)$ and manufacturing (14.9\%). The majority of companies (89.4\%) operated fully or partially within the domestic market. Moreover, they were mainly under the management of the entrepreneur (64.2\%). About the attitude towards a new hiring, $55,2 \%$ of the firms kept the same number of employees during last 3 years, while $33,3 \%$ increased their workforce, about the future more than $70 \%$ of the companies predict to hire a new resource.

\section{The figure of Administrative Clerk}

The job position "Administrative Clerk" is referring to who accomplishes and supports all the aspects of planning, organization, administration and management in the firm performing not-directorial tasks.

Here in the following list, some of principal tasks for Administrative Clerk are presented:

- managing and taking part to meeting and conference 
Table 1 Frequency distribution for Electus Data

\begin{tabular}{|r|r|r|}
\hline N of employees & Activity sectors & Activity market \\
\hline $0-19(37.5 \%)$ & Service industries $(62.1 \%)$ & Both $(45.7 \%)$ \\
$20-49(14.5 \%)$ & Personal services $(16.2 \%)$ & Domestic $(43.8 \%)$ \\
$50-249(25.6 \%)$ & Manufacturing $(14.9 \%)$ & International $(10.6 \%)$ \\
$250+(22.4 \%)$ & Other $(6.8 \%)$ & \\
\hline Company Supervisor & Resources in last 3 years & Hirings in the future \\
\hline Entrepreneur (64.2\%) & More $(33.3 \%)$ & Yes $(70.8 \%)$ \\
Manager (25.4\%) & Equal $(55.2 \%)$ & \\
Other $(10.3 \%)$ & Less $(5.5 \%)$ & \\
& No answer $(6.0 \%)$ & \\
\hline
\end{tabular}

- arranging and archiving documents, procedures and provisions

- controlling the respect of rules and procedures

- accomplishing fulfillments

- promulgating authorizations for the payment

- acquiring and spreading information

- collecting data for statistical purposes

The Administrative Clerk must possess skills, capabilities and competencies related to multiple fields. First of all, these attitudes have to be related with the accounting and economic world. Secondly, they have to manage basics of Law, in fact their activity deal with bureaucratic part of the business process, that is to say legal procedures and redaction of documents and fulfillments. Thirdly, they have to be familiar with business management knowing fundamentals and methods of the business related to strategical planning and resource allocation. Finally, they need to have also knowledge of Computer Sciences for the use of office tools as computers, e-mail, internet and specific programs [8].

The Administrative Clerk figure belongs to the superior class of "general affair and secretary employed" and in the period 2015-2019, for this class, it is predicted an increasing of employment of $2.6 \%$. The occupational base should increase of 32.300 units. The labour demand should amount to 545.510 units. In particular, it is predicted stability in trend variation (between $-2 \%$ and $+2 \%)[8]$.

Moreover, for 2016 year, 15090 new hirings are predicted for Administrative Clerk, $46 \%$ in permanent contract and $39 \%$ in medium-sized and large companies. According this predictions, $74 \%$ of this hirings will concern the high-school graduated and 25\% people with academic studies and in the $67 \%$ of cases there will be no distinction between males and females [21].

\section{Latent Class Conjoint Analysis}

Conjoint Analysis (CA) is a statistical technique commonly used in market research to assess how features or attributes of a product or service influence individuals' preferences [7]. In CA, a subset of such attributes, usually those 
considered as more relevant, are combined within an experimental design in order to define a set of potential product or service profiles. Each of such hypothetical profiles are then shown to individuals who express their preference in terms of a numeric rating scale or by indicating their favorite profile. Using $\mathrm{CA}$, it is possible to determine which combination of attributes is most influential and to derive the utilities associated to specific levels of such attributes, also known as partial utilities or part-worths. One of the major uses of CA is for segmentation analysis [6,24] with the aim of identifying groups of individuals characterized by homogeneous utility functions or preferences. In this study CA is carried out to evaluate which characteristics of a graduate's profile employers prefer for a potential candidate in the job position of administrative clerk. In particular, in order to detect if there exist unobserved subgroups of employers having homogeneous preferences about graduates' characteristics for this position, a Latent Class Metric Conjoint Analysis [3] is employed. The LCMCA is a model-based approach in which model parameters and subgroups (segments or latent classes) are estimated simultaneously, and segments are composed of individuals whose regression coefficients (or conjoint part-worth utilities) are similar. In literature, other more traditional post-hoc segmentation techniques are known, for example the tandem approach [6]. Following this technique, at first a conjoint model is estimated for each respondent and then individual level part-worth estimates (or partial utilities) are used as input data for cluster analysis to obtain homogeneous subgroups [15]. The second stage of a post-hoc clustering could be performed using the hierarchical clustering $[14,16]$ and non-hierarchical clustering [6]. In general the tandem approach is effected by the same problems affecting clustering approach: different clustering methods often yield different results, in terms of number of clusters and their composition. Moreover in presence of highly fractionated designs individual-level part-worth estimates are rather unstable and then may be untrustworthy when employed in successive clustering algorithms [24].

LCMCA is a statistical modelling technique included in the more general class of Finite Mixture Models (FMMs) [12]. FMMs assume that the population is composed by an unknown but finite number of unobserved subpopulations or latent classes, each characterized by its own distinct parametric distribution of the response variable. In such circumstances, each observation is supposed to belong to one of these latent classes, although it is not known in advance from which a particular observation arises, and the use of classical regression model is inadequate since a single set of coefficients is estimated for all observations. Following the FMMs approach, LCMCA relaxes the single homogeneous population assumption to allow for parameter differences across $G$ latent classes and supposes that the marginal density function of the response variable y is given by a weighted sum over the $G$ mixture components, with weights indicating the a-priori probability for an observation to come from a specific component [3]: 


$$
f\left(y_{i j} \mid \pi, x, z, \Sigma\right)=\sum_{g=1}^{G} \pi_{g \mid z} f_{g}\left(y_{i j} \mid x, z, \beta_{g} \Sigma_{g}\right)
$$

where $y_{i j}$ is the vector of response variable which refers to the rating expressed by employer $i$ to conjoint profile $j ; \pi=\left(\pi_{1}, \pi_{2}, \ldots, \pi_{G-1}\right)$ are $G-1$ independent mixing proportions of the mixture, such that $0 \leq \pi_{g} \leq 1 ; x$ is a matrix containing the $M$ conjoint dummy variables which defines the profiles evaluated; $\beta_{g}$ is the vector of the estimated conjoint part-worth coefficients for subgroup $g$, and $\Sigma=\left(\Sigma_{1} \Sigma_{2}, \ldots, \Sigma_{g}\right)$ is $J \times J$ vector of covariance matrices of the error terms estimated for each subgroup. Moreover, given that the weights depend on a set of explanatory variables, also referred to as concomitant variables [25], $z$ defines the vector of variables which characterise employers. In particular, each of the conditional distributions, $f_{g}$, is specified as a conditional multivariate distribution, as it follows:

$$
f_{g}\left(y_{i j} \mid, x, z, \beta_{g}, \Sigma_{g}\right)=(2 \pi)^{-J / 2}\left|\Sigma_{g}\right|^{-1 / 2} \exp \left[\frac{1}{2}\left(y_{i j}-x \beta_{g}^{\prime}\right) \Sigma_{g}^{-1}\left(y_{i j}-x \beta_{g}^{\prime}\right)^{\prime}\right]
$$

Given the metric response variable, each of the conditional distributions, $f_{g}$, is conventionally specified as a conditional multivariate normal distribution. Instead, the prior probability of group membership varies as a multinomial logistic regression model, in function of the concomitant variables, as it follows:

$$
\pi_{k \mid z}=\frac{\exp \left(\gamma_{0 g}+z \gamma_{1 g}\right)}{\sum_{g=1}^{G} \exp \left(\gamma_{0 g}+z \gamma_{1 g}\right)}
$$

where $\gamma_{0 g}$ is the intercept while $\gamma_{1 g}$ contains the vector of regression coefficients, quantifying the effect of the concomitant variables on the prior probability for class $g$. For identification purpose, usually $\gamma_{01}=0$ and $\gamma_{11}=0$, and designate the first category as a reference class. Moreover, the following constraints hold:

$$
\sum_{g=1}^{G} \pi_{g \mid z}=1, \pi_{g \mid z}>0
$$

Once the estimates of all the model parameters of the mixture of probability density are obtained, the posterior probability that an observation belongs to class $g$, denoted with $p_{i g}$, can be calculated by updating the previous according to the Bayes' theorem, as follows:

$$
\hat{p}_{i g}=\frac{\hat{\pi}_{g \mid z} \hat{f}_{i g}\left(y_{i j} \mid x, z, \beta_{g} \Sigma_{g}\right)}{\sum_{g=1}^{G} \hat{\pi}_{g \mid z} \hat{f}_{i g}\left(y_{i j} \mid x, z, \beta_{g} \Sigma_{g}\right)}
$$

where $\sum_{g=1}^{G} \hat{p}_{i g}=1$ and $0 \leq \hat{p}_{i g} \leq 1$. 
The posterior probabilities provide a probabilistic allocation of observations to the latent classes and can be used to classify data by assigning each employer to the class with the maximum posterior probability. Parameter estimation was carried out via Maximum Likelihood (ML) by using the Expectation-Maximization (E-M) algorithm [2], in which conjoint part-worth coefficients and class membership are obtained simultaneously (see, for more details [3]). The conventional Akaike's information criterion (AIC) [1] was used for choosing the number of latent classes.

\section{Application and Results}

The results of the LCMCA are summarised in Table 2, Table 3 and Table 4 , respectively. Table 2 shows the summary statistics after estimating several LCMCAs, starting from the aggregate solution, with one class $(G=1)$, to the most complex one, with five latent classes $(G=5)$. The corresponding values of Log-likelihood, degree of freedom (DF) and AIC were compared for determining the number of classes to retain. Specifically, based on the information criteria in Table 2, the solution with $G=3$ seems to represent the latent structure underlying the employers' ratings quite well. In fact, although the solution with $G=5$ classes is the one with the lowest AIC, its value is not much lower than that of the more parsimonious three-class solution. The number of classes at this point meets also the 'elbow criterion' as showed in Figure 1, since graphing the AIC values versus the number of classes, the gain in model fit is higher with the addition of the third class but, as the number of classes increases, the marginal gain drops, resulting in a pronounced angle or 'elbow' in the plot. Therefore, $G=3$ seems to be the 'best' model with the fewest number of classes that has an adequate overall goodness of fit.



Fig. 1 Elbow criterion for the choice of number of groups G 
Table 2 Latent class solution

\begin{tabular}{cccc}
\hline G & Log-likelihood & DF & AIC \\
\hline 1 & -636.5632 & 19 & 1311.126 \\
2 & -603.9745 & 48 & 1303.949 \\
3 & -531.3041 & 76 & 1214.608 \\
4 & -487.0319 & 104 & 1182.064 \\
5 & -454.2653 & 132 & 1172.531 \\
\hline
\end{tabular}

Table 4 provides the estimated regression coefficients (or part-worths) for the $G=3$ latent classes solution. Coefficients significantly different from zero at $p<0.05$ and $p<0.01$ are indicated with superscript $(*)$ and $(* *)$, respectively. Given that categorical attributes were preventively converted into the appropriate number of dummy variables (i.e. one less than the number of levels of the attribute), the intercept represents the average rating of the reference profile and refers to a graduate in foreign languages, with a Master's degree, a low final grades, with a knowledge of English language, no working experience, and not willing to business trips. At a first glance, it is clear the substantial difference between the aggregate part-worth coefficients and those in each sub-model of the three-class solution. Moreover, the aggregate model is also the one with the highest value of AIC and this confirms that a single set of regression coefficients estimated for all the employers may produce misleading results. The first class is the one with the lowest average rating corresponding to the reference profile (2.56) and identifies especially Economics as the most preferred degree, whereas Law, Statistics and Engineering are also appreciated but to a lesser extent. Among the employers included in this class, it seems preferable for a candidate to have some kind of work experience, excluding the internship. On the other hand, low final grades and willing to long-term business trips produce a lower preference. The second class is the one with the higher average rating (8.25) and this indicates that the reference profile is already highly appreciated. Given such a high average score, part-worth coefficients are almost all negative. In particular, employers within such class evaluate Economics, Engineering, Mathematics and computer sciences as less important degrees. On the contrary, a Bachelor's degree and a medium final grades increase employers' preference. The third class is the one with the intermediate average rating and its value is also very similar to that of the aggregate model (4.46). Mathematics and computer sciences and Economics are the most preferred degrees. Political science is also evaluated positively but to a lesser extent. For employers in this class, a previous work experience both as a stable experience and internship experience is relevant.

Table 4 shows the Maximum likelihood estimates of the multinomial logistic regression model that allows to determine which variables affect the latent classes' membership. Here, Class 1 has been defined as the reference outcome. Among the available variables which describe the characteristics and the context of the company, only the variables 'Recruitment of staff within one year', 'Company run by a manager' and 'Company committed also in the foreign market' seem to affect class membership when using the usual 0.05 as signif- 
Table 3 Maximum likelihood estimates of part-worth coefficients for each latent class

\begin{tabular}{|c|c|c|c|c|}
\hline & & Latent & class & \\
\hline & 1 & 2 & 3 & Aggregate \\
\hline & $n_{1}=79$ & $n_{2}=51$ & $n_{3}=169$ & $\mathrm{n}=299$ \\
\hline Intercept & $2.56^{* *}$ & $8.25^{* *}$ & $4.46^{* *}$ & $4.79^{* *}$ \\
\hline Philosophy and Literature & $2.60 * *$ & $-1.65 * *$ & -2.20 & -0.88 \\
\hline Education sciences & $1.54^{*}$ & -0.02 & -0.48 & 0.15 \\
\hline Political sciences & 1.21 & $-1.23^{* *}$ & $1.45^{* *}$ & 0.97 \\
\hline Economics & $6.52^{* *}$ & $-5.21 * *$ & $2.18^{* *}$ & $1.92^{* *}$ \\
\hline Law & $4.29 * *$ & $-3.75^{* *}$ & -0.47 & -0.25 \\
\hline Statistics & $3.99 * *$ & $-2.77^{* *}$ & -0.63 & 0.42 \\
\hline Engineering & $3.81^{* *}$ & $-4.74 * *$ & $-2.25 * *$ & $-1.16^{*}$ \\
\hline Mathematics and computer sciences & $2.68^{* *}$ & $-5.41^{* *}$ & $2.92^{* *}$ & 0.45 \\
\hline Psychology & $2.80 * *$ & $-3.66^{* *}$ & $-2.42^{* *}$ & $-1.61^{*}$ \\
\hline Bachelor's degree & -0.23 & $0.59 * *$ & 0.47 & 0.49 \\
\hline Low final grades & $-1.62^{* *}$ & $-1.13^{* *}$ & $-1.01 * *$ & $-1.25^{* *}$ \\
\hline Medium final grades & $-0.92^{*}$ & $1.70^{* *}$ & $-0.94 * *$ & -0.50 \\
\hline No knowledge of English language & 0.49 & $-0.75 * *$ & $-1.57 * *$ & $-0.87 * *$ \\
\hline Internship experience & -0.56 & $0.84^{* *}$ & $1.37^{* *}$ & 0.46 \\
\hline Occasional working experience & $2.10 * *$ & $-0.25 * *$ & 0.82 & 0.66 \\
\hline Stable working experience & $1.04^{*}$ & $-2.28 * *$ & $2.36^{* *}$ & $1.07^{* *}$ \\
\hline Willing to short-term business travels & -0.44 & $-1.46^{* *}$ & $0.85 * *$ & -0.15 \\
\hline Willing to long-term business travels & $-1.33^{*}$ & $-0.88 * *$ & 0.53 & -0.10 \\
\hline
\end{tabular}

icance level. However, considering 0.10 as significance level also the variables 'Hired personnel over the past 3 years' and 'Education of the last administrative hired graduated' contribute to explain class membership. In particular, the probability of being in Class 2 is higher for companies which plan to recruit staff within one year $(p=0.007)$ but lower for those that hired personnel over the past 3 years $(p=0.070)$ and those which have already taken a graduated as administrative $(p=0.076)$. On the other hand, the probability of being in Class 3 is higher for companies which plan to recruit staff within one year $(p<0.0001)$, for companies run by a manager $(p=0.036)$ and for companies committed also in the foreign market $(p=0.033)$ but lower for those that hired personnel over the past 3 years $(p=0.072)$.

Looking at the table 4 , it seems adequate to identify the three latent groups by their peculiar features. In particular, the first group ( $26.4 \%$ of the sample) is characterized by companies run by not a managerial view, working in a service sector in prevalence in domestic market, they neither will do recruitment new staff in the next year neither hired personnel over the past three years. This group could be named Domestic Consolidated Companies. The best profile for the $\mathrm{AC}$ required by these companies results to be related to a classical view of the position: a well graduated in Economics with some kind of working experience.

The second group ( $17.1 \%$ of the sample) is composed by big sized companies, they will recruit staff in the next year, but they did not hire personnel over the past three years. This group could be called as Static Companies: respect to the expected profile of new graduates they seem to prefer new graduates with a not suitable major for AC (Language) with Bachelor's degree and 
Table 4 Maximum likelihood estimates of latent class membership probability

\begin{tabular}{lrrr}
\hline & Estimate & $\mathrm{SE}$ & $\mathrm{p}$ \\
\hline Class 1 (base outcome) & & & \\
\hline Class 2 & -2.42 & 0.77 & 0.002 \\
Intercept & -1.14 & 0.63 & 0.070 \\
Hired personnel over the past 3 years & 1.00 & 0.56 & 0.076 \\
Education of the last administrative hired: graduated & 0.89 & 0.61 & 0.147 \\
Company run by a manager & 0.96 & 0.68 & 0.161 \\
Company with 20 or more employees & -0.72 & 0.54 & 0.182 \\
Company in the services sector & 0.52 & 0.53 & 0.319 \\
Company committed also in the foreign market & 1.74 & 0.65 & 0.007 \\
Recruitment of staff within one year & & & \\
\hline Class 3 & -1.42 & 0.77 & 0.066 \\
Intercept & -1.08 & 0.60 & 0.072 \\
Hired personnel over the past 3 years & 0.21 & 0.53 & 0.685 \\
Education of the last administrative hired: graduated & 1.30 & 0.62 & 0.036 \\
Company run by a manager & -0.30 & 0.60 & 0.617 \\
Company with 20 or more employees & -0.52 & 0.53 & 0.321 \\
Company in the services sector & 1.05 & 0.49 & 0.033 \\
Company committed also in the foreign market & 3.19 & 0.76 & 0.000 \\
\hline Recruitment of staff within one year & & & \\
\hline
\end{tabular}

a medium final grade, the English knowledge and working experience are not required. In some way, they prefer new graduates who must be fully trained.

The third group ( $56.5 \%$ of the sample) is represented by small or medium enterprises, run by a manager and committed also in the foreign market with a willingness to recruit new staff in the next year. They can be named as Dynamic Companies. The new graduate profile fits the description of these firms: it has to be a student in Economics or Political Sciences major, with a higher final grade and an English knowledge suitable to communicate with foreign people. Important requirements are also working experience and willing to business trips.

\section{Discussion and final remarks}

The unemployment could be considered a driven sign about economic crisis that makes weaker the labour market in particular for young people with less work experience, as for example the new graduated students that face up with the difficulties of a new job. An important strategy of the Horizon 2020 program is to consider and reduce the mismatch between the demand and offer in labour market for the integration of young people in the labour force. The universities could try to be the connection between the job market and the students. The results of this research aim to improve learning and employability policies within the academia and would like to explain the relationships between the enterprises and universities, with reference to the labour market for the new graduates. The novelty of the paper is represented by the search of homogeneous groups of entrepreneurs respect to the expected features of the new graduates for a job. Using the survey ELECTUS, a segmentation of 
employers' preferences for graduates' profiles for administrative clerk is carried out by using a Latent Class Metric Conjoint Analysis. Specifically, the analysis detects the existence of three unobserved subgroups of employers having homogeneous preferences about graduates' characteristics. The benefit to use this methodology is given by the substantial difference between the aggregate part-worth coefficients in conjoint analysis and those in each sub-model of the three-class solution: often, in fact, a single set of regression coefficients estimated for all the employers may produce misleading results, as underlined by the results. In general the features of the profiles for the new graduates job are very different for every sub-groups but all respondents agree that a low final grades at graduation is not a preferable. Certainly the characteristics of the companies could influence the preferences about graduates' characteristics: the membership of the latent groups seems in fact to be effected by peculiar factors. In fact, the Domestic Consolidated Companies, run by not a managerial view, working in a service sector in prevalence in domestic market, without willingness to recruit in the past and in the future, require a well graduated students in Economics with some kind of working experience for AC position. The Static Companies, composed by big sized companies with the willingness to recruit in the future but not in the past, prefer new graduates who must be fully trained. The Dynamic Companies, represented by SME run by a manager and committed also in the foreign market with a willingness to recruit new staff in the next year, look for a new graduate in Economics or Political Sciences major, with a higher final grade and an English knowledge suitable to communicate with foreign people with working experience and willing to business trips.

Future works are devoted in two directions. The first one is represented by the comparisons of the results of LCMCA for the five job positions proposed by ELECTUS survey, while the second one regards the comparison of the results of LCMCA with the results of the segmenting using the tandem approach. 


\section{References}

1. Akaike, H.: Information theory and an extension of the maximum likelihood principle. In: Petrov, N., and Csadki, E. (eds.) Proceedings of the Second International Symposium of Information Theory, pp. 267281. Budapest: Akademiai Kiado (1973)

2. Dempster, A., Laird, N., Rubin, D.: Maximum Likelihood from Incomplete Data via the EM-Alogrithm. Journal of the Royal Statistical Society: Series B, 39(1), 1-38 (1977).

3. DeSarbo, W.S, Wedel, M.A., Vriens, M., Ramaswamy, V.: Latent Class Metric Conjoint Analysis. Marketing Letters, 3(2), 137-288 (1992).

4. European Commission. Communication from the commission to the european parliament, the council, the european economic and social committee and the committee of the regions. In Official Journal of the European Union, 1161: 1-43 (2010).

5. Fabbris, L. and Scioni, M. Dimensionality of scores obtained with a paired-comparison tournament system of questionnaire item. In A. Meerman and T. Kliewe, eds., Academic Proceedings of the 2015 University-Industry Interaction Conference: Challenges and Solutions for Fostering Entrepreneurial Universities and Collaborative Innovation (2015).

6. Green, P.E., Krieger, A.M.: Segmenting Markets with Conjoint Analysis. Journal of Marketing, 55(4), 20-31 (1991).

7. Green, P.E., and Rao, V.R. (1971). Conjoint Measurement for Quantifying Judgmental Data. Journal of Marketing Research, 8(3), 355-363.

8. Isfol. Ministero del lavoro - classificatore delle professioni. URL http: //fabbisogni.isfol.it (2017).

9. Istat, http://www.istat.it/it/lavoro-e-retribuzioni (2017).

10. Louviere, J.J., Hensher, D.A., Swait, J.D.: Stated Choice Methods. Analysis and Application. Cambridge University Press (2000)

11. Lindeboom, M., Van Ours, J., and Renes, G. Matching employers and workers: an empirical analysis on the effectiveness of search. In Oxford economic papers, 45-67 (1994).

12. McLachlan, G. J., Peel, D.: Finite Mixture Models. New York: Wiley (2000)

13. Charrad, M., Ghazzali, N., Boiteau, V., Niknafs A.: NbClust: An R Package for Determining the Relevant Number of Clusters in a Data Set. In Journal of Software Statistics, $61(6), 1-36(2014)$

14. Punj, G. and Stewart D.W. Cluster Analysis in Marketing Research: Review and Suggestions for Application. In Journal of Marketing Research, 20(2): 134-148 (1983)

15. Ramaswamy, V, Cohen, S.E.: Latent Class Models for Conjoint Analysis. In: Gustafsson, A., Herrmann, A., Huber, F. (eds.) Conjoint Measurement -Theory and Applications (4th edition), pp. 295-319. Springer Berlin Heidelberg (2007).

16. Romano, E., Lauro, C. and Giordano, G. An Inter-model distance for clustering utility functions. Statistica Applicata - Italian Journal of Applied Statistics. 18: 521-533 (2006)

17. Russo, G., Gorter, C., Nijkamp, P., and Rietveld, P. Employers recruitment behaviour: an empirical analysis of the role of personnel management attitudes. In Labour, 11 (3): 599-623 (1997).

18. Sawtooth, S. URL http://www.sawtoothsoftware.com (2017).

19. Tanveer Choudhry, M., Marelli, E., and Signorelli, M. Youth unemployment rate and impact of financial crises. In International journal of manpower, 33 (1): 76-95 (2012).

20. Taylor, M.S. and Bergmann, T.J. Organizational recruitment activities and applicants reactions at different stages of the recruitment process. In Personnel Psychology, 40 (2): 261-285. (1987).

21. Unioncamere. Sistema informativo excelsior. In Progetto Excelsior. Sintesi dei principali risultati. URL http://excelsior.unioncamere.net (2017).

22. Vriens, M., Wedel, M.A., Wilms, T.: Metric Conjoint Segmentation Methods: A Monte Carlo comparison. Journal of Marketing Research, 33(1), 73-85 (1996).

23. Wedel, M.A.: Concomitant variables in finite mixture models. Statistica Neerlandica, $56(3), 362-375$ (2002).

24. Wedel, M.A., Kistemaker, C.: Consumer Benefit Segmentation using Clusterwise Linear Regression. International Journal of Research in Marketing, 6(1), 295-319 (1989).

25. Wedel, M., DeSarbo, W. S.: Mixture Regression Models. In: Hagenaars, J. A., and McCutcheon, A. L. (eds.). Applied Latent Class Analysis, pp. 366-382. Cambridge (2002)

26. Wedel, M., Kamakura, W. A.: Market Segmentation - Conceptual and Methodological Foundations. Kluwer Academic Publishers, Boston, MA, USA, 2nd edn (2000). 